\title{
Expression of intestinal transporter genes in beagle dogs
}

\author{
SOO-MIN CHO ${ }^{1 *}$, SUNG-WON PARK $^{1,2^{*}}$, NA-HYUN KIM ${ }^{1}$, JIN-A PARK $^{1}$, HEE YI $^{1}$, \\ HEE-JUNG CHO ${ }^{1}$, KI-HWAN PARK ${ }^{3}$, INGYUN HWANG ${ }^{4}$ and HO-CHUL SHIN ${ }^{1}$
${ }^{1}$ Department of Veterinary Pharmacology and Toxicology, College of Veterinary Medicine, Konkuk University, Seoul 143-701;
${ }^{2}$ Toxicology and Chemistry Division, Animal Plant and Fisheries Quarantine and Inspection Agency, Anyang 430-824; ${ }^{3}$ Department of Food Science \& Technology, Chung-Ang University, Ansung 456-756; ${ }^{4}$ Food Chemical Residues Division, \\ Korea Food \& Drug Administration, Osong 363-951, Republic of Korea
}

Received August 8, 2012; Accepted October 12, 2012

DOI: $10.3892 /$ etm.2012.777

\begin{abstract}
This study was performed to produce a transcriptional database of the intestinal transporters of beagle dogs. Total RNA was isolated from the duodenum and the expression of various mRNAs was measured using GeneChip ${ }^{\circledR}$ oligonucleotide arrays. A total of 124 transporter genes were detected. Genes for fatty acid, peptide, amino acid and glucose and multidrug resistance/multidrug resistance-associated protein (MDR/MRP) transport were expressed at relatively higher levels than the other transporter types. The dogs exhibited abundant mRNA expression of the fatty acid transporters (fatty acid binding proteins, FABPs) FABP1 and FABP2, the ATP-binding cassettes (ABCs) ABCB1A and ABCC2, the amino acid/peptide transporters SLC3A1 and SLC15A1, the glucose transporters SLC5A1, SLC2A2 and SLC2A5, the organic anion transporter SLC22A9 and the phosphate transporters SLC20A1 and SLC37A4. In mice, a similar profile was observed with high expression of the glucose transporters SLC5A1 and SLC2As, the fatty acid transporters FABP1 and FABP2, the MDR/MRP transporters ABCB1A and ABCC2 and the phosphate transporter SLC37A4. However, the overall data reveal diverse transcriptomic profiles of the intestinal transporters of dogs and mice. Therefore, the current database may be useful for comparing the intestinal transport systems of dogs with those of mice to better evaluate xenobiotics.
\end{abstract}

\section{Introduction}

Intestinal drug transporters have great potential for drug absorption $(1,2)$. They may serve as either drug targets or drug delivery systems. It is generally assumed that at least

Correspondence to: Professor Ho-Chul Shin, Department of Veterinary Pharmacology and Toxicology, College of Veterinary Medicine, Konkuk University, 1 Hwayang-dong, Gwangjin-ku, Seoul 143-701, Republic of Korea

E-mail: hshin@konkuk.ac.kr

*Contributed equally

Key words: gene expression, transporters, intestine, $\operatorname{dog}$
$5 \%$ of all human genes are associated with transporters which is consistent with the biological significance of transporters and their roles in cell homeostasis. The identification and characterization of drug transporters has provided a scientific basis for understanding drug delivery and disposition, the molecular mechanisms of drug interactions and inter-individual/inter-species differences (3). Various types of xenobiotic or drug transporters have been identified as being important as barriers against toxic compounds and influx pumps to take up nutrients into the body. Since these xenobiotic transporters generally have a wide range of recognition specificities and accept various types of compounds as substrates, the localization and functional expression of such transporters may be a critical factor in the disposition and subsequent biological activity of therapeutic agents.

Dogs have emerged as a primary species for the study of biology and human diseases (4). The organization of the dog genome has been studied extensively in the last ten years. With advances in genomics, microarray technology has been used to identify tissue-specific genes, including intestinal transporters. It is now accepted that the process of drug absorption in the intestine is highly associated with the functional expression of intestinal transporters (5). The current study was therefore carried out to generate a gene expression database of transporters in the canine duodenum.

\section{Materials and methods}

Materials. The TRIzol ${ }^{\circledR}$ reagent and SuperScript Choice System cDNA synthesis kit were purchased from Invitrogen (Carlsbad, CA, USA). The BioArray high-yield RNA transcript labeling kit was obtained from Enzo Biochem (New York, NY, USA). The RNeasy kit was supplied by Qiagen (Valencia, CA, USA). The Canine 2.0 and Mouse 430A 2.0 GeneChips were provided by Affymetrix (Santa Clara, CA, USA). GeneChip hybridization and scanning were performed at the Seoulin Molecular Biology Technique Center (Seoul, Korea).

Animals. Beagle dogs (4 males, 5.5-7.6 kg; Marshall BioResource, Beijing, China) were housed in a controlled semi-barrier system room at Chemon Co. (Yongin, Korea). The animals were considered to be healthy based on clinical examination (Korea Food and Drug Administration Guide for 
the Care and Use of Laboratory Animals, 2009). ICR mice (30-40 g) were obtained from Orient Bio Co. (Seoul, Korea) and housed in a controlled animal room at Konkuk University (Seoul, Korea). The animals were fed solid pellets and provided with water ad libitum. All procedures were approved by the Konkuk University Institutional Animal Care and Use Committee.

RNA isolation. Mucosal tissues obtained from dog and mouse duodenums were immediately scraped with a clean glass slide, transferred to a new frozen vial and dipped into liquid $\mathrm{N}_{2}$. Tissue ( $\left.100 \mathrm{mg}\right)$ was added to $1 \mathrm{ml}$ TRIzol reagent and homogenized with a razor on ice. The homogenate was transferred to a new tube and then $200 \mu \mathrm{l}$ chloroform was added to the TRIzol mixture. Following centrifugation at $12,500 \mathrm{rpm}$ for $15 \mathrm{~min}$ at $4^{\circ} \mathrm{C}$, the aqueous phase was transferred to a new tube. The RNA was then precipitated with $500 \mu$ l isopropanol and washed with $80 \%$ ethanol. The RNA was further purified with an RNeasy Mini kit (Qiagen) according to the manufacturer's instructions. The concentration of the purified RNA was measured at $260 \mathrm{~nm}$ and $5 \mu \mathrm{g}$ purified RNA was mixed with RNA loading buffer and heated at $75^{\circ} \mathrm{C}$ for $15 \mathrm{~min}$. After cooling on ice for $5 \mathrm{~min}$, the RNA was loaded onto $1 \%$ agarose/formaldehyde gel in $1 \mathrm{X}$ MOPS buffer. The gel was run at $80-100 \mathrm{~V}$ for $50 \mathrm{~min}$ and the presence of two sharp 18S and 28S bands was confirmed under UV light.

Microarray assay. Once the total RNA samples were prepared, probe synthesis, hybridization, detection and scanning were performed according to the standard instructions of the manufacturer (Affymetrix, Inc.). The cDNA was synthesized using the One-Cycle cDNA Synthesis kit. Single-stranded cDNA was synthesized using Superscript II reverse transcriptase and T7-oligo(dT) primers at $42^{\circ} \mathrm{C}$ for $1 \mathrm{~h}$. Double-stranded (ds) cDNA was obtained through a reaction using DNA ligase, DNA polymerase I and RNase $\mathrm{H}$ at $16^{\circ} \mathrm{C}$ for $2 \mathrm{~h}$, followed by T4 DNA polymerase at $16^{\circ} \mathrm{C}$ for $5 \mathrm{~min}$. After clean up with a Sample Cleanup Module (Affymetrix, Inc.), ds-cDNA was used for in vitro transcription (IVT). cDNA was transcribed using the GeneChip IVT Labeling kit (Affymetrix, Inc.) in the presence of biotin-labeled CTP and UTP. The resulting biotin-labeled IVT-RNA was again purified with a Sample Cleanup Module (Affymetrix, Inc.) and subsequently fragmented. Fragmented cRNA was hybridized at $45^{\circ} \mathrm{C}$ for $16 \mathrm{~h}$ according to the manufacturer's instructions. Following hybridization, the arrays were washed in a GeneChip Fluidics Station 450 with a non-stringent wash buffer at $25^{\circ} \mathrm{C}$ and then by a stringent wash buffer at $50^{\circ} \mathrm{C}$. The arrays were then stained with a streptavidin-phycoerythrin complex. After staining, the intensities were determined with a GeneChip scanner. The duodenal mRNA expression profile obtained from microarray data analyses for SLC15A1 was validated using semiquantitative RT-PCR. The RT-PCR assay was performed as described previously (6). The pattern of SLC15A1 mRNA expression in the individual biopsies determined by RT-PCR was similar to that observed in the microarray data.

Data analysis. Official symbols and gene names were used in accordance with the symbol and name lists approved by the Human Genome Organization (HUGO) Gene Nomenclature
Committee (http://www.genenames.org). Data analysis was performed using GeneSpring 7.2 software (Silicon Genetics, Redwood City, CA, USA). The numeric data were extracted from DAT images and normalized using Microarray Suite software. Gene function analysis was performed using the gene ontology-mining tool of NetAffx, which is based on the Gene Ontology database (http://www.geneontology.org). GeneSpring also uses data from public genomics databases to build gene ontologies based on annotation information. For the present GeneChip probe array study, the data for each gene represented data from 11-20 probe pairs, each $\sim 25$ bp in length. The overall target-specific intensity was measured as the difference between the intensity of the perfectly matched and mismatched probes. For normalization, data from each expression array were scaled so that the overall fluorescence intensity across each chip was equivalent (average target intensity set at 500). The One-Sided Wilcoxon Signed Rank test was employed to generate the detection P-value. If the overall intensity of the perfect match was significantly larger than that of the mismatch, the detection P-value was small. The probed gene set was considered to be present if the P-value was $<0.04$. If the P-value was $>0.06$, the probe set was considered to be absent. The change algorithm generated a change P-value and an associated fold-change value. The second algorithm gave a quantitative estimate of the change in gene expression in the form of a signal log ratio. The level of gene expression was considered to be increased if its change P-value was $<0.0025$ and the gene expression was considered to be decreased if its change P-value was $>0.9975$.

\section{Results}

Sequence analysis. A total of 43,035 sequences from the dog duodenum were analyzed and $60 \%$ exhibited $>1$-fold changes. As shown in Table I, the total numbers of detected transporter genes were 124 in the dog duodenum and 130 in the mouse intestine. Among the transporter groups, the expression levels of fatty acid, peptide, amino acid, glucose and multidrug resistance/multidrug resistance-associated protein (MDR/MRP) transporter genes were relatively higher than those of other transporter gene groups in the two species.

Glucose transporters. Fig. 1 shows the similar expression profiles of the glucose transporter genes in the dogs and mice. The majority of the SLC2 family (facilitated glucose transporters, GLUTs) and SLC5 family (sodium/glucose cotransporters, SGLTs) genes were expressed. SLC2A2, SLC2A5 and SLC5A1 were the dominantly expressed genes in the two species.

Amino acid and peptide transporters. Fig. 2 shows the expression levels of various amino acid and peptide transporter genes in the duodenums of the dogs and mice. SLC3A1 and SLC15A1 were the dominantly expressed transporters in dogs, while SLC7A9 and SLC7A7 were highly expressed in mice. The expression of SLC15A1 was $>15$-fold higher in dogs than in mice $(\mathrm{P}<0.01)$.

Fatty acid transporters. The expression levels of fatty acid transporter genes are shown in Fig. 3. The overall expression 
Table I. Numbers of transporter genes expressed in dog and mouse intestines.

\begin{tabular}{|c|c|c|c|c|}
\hline \multirow[b]{2}{*}{ Transporter cluster } & \multicolumn{2}{|c|}{ Dog } & \multicolumn{2}{|c|}{ Mouse } \\
\hline & Number & $\begin{array}{c}\text { Expression level } \\
(\text { mean } \pm \mathrm{SD})\end{array}$ & Number & $\begin{array}{l}\text { Expression level } \\
(\text { mean } \pm \mathrm{SD})\end{array}$ \\
\hline Glucose transporters & 18 & $8.1 \pm 12.8$ & 11 & $19.5 \pm 37.0$ \\
\hline Amino acid transporters & 7 & $12.6 \pm 31.9$ & 15 & $7.1 \pm 12.7$ \\
\hline Peptide transporters & 4 & $46.3 \pm 62.1$ & 3 & $25.8 \pm 37.5$ \\
\hline Fatty acid transporter & 12 & $75.0 \pm 167.8$ & 13 & $41.9 \pm 85.0$ \\
\hline Mitochondrial solute carrier & 10 & $16.6 \pm 50.5$ & 16 & $23.8 \pm 42.3$ \\
\hline Nucleoside transporters & 5 & $1.7 \pm 0.8$ & 6 & $3.6 \pm 3.4$ \\
\hline Nucleobase transporter & 2 & $1.3 \pm 1.1$ & 3 & $3.7 \pm 7.6$ \\
\hline MDRs/MRPs & 7 & $10.8 \pm 17.3$ & 17 & $4.5 \pm 7.1$ \\
\hline Organic anion transporters & 13 & $1.7 \pm 2.9$ & 14 & $0.8 \pm 1.5$ \\
\hline Organic cation transporters & 8 & $1.3 \pm 1.2$ & 9 & $3.7 \pm 4.5$ \\
\hline Phosphate transporters & 11 & $3.5 \pm 3.8$ & 5 & $5.2 \pm 5.56$ \\
\hline Sodium/hydrogen exchangers & 8 & $1.0 \pm 0.8$ & 2 & $1.8 \pm 0.5$ \\
\hline Sulfate transporter & 2 & $6.1 \pm 7.3$ & 2 & $1.6 \pm 1.1$ \\
\hline Zinc Transporters & 17 & $2.9 \pm 4.2$ & 14 & $6.7 \pm 5.2$ \\
\hline Total & 124 & - & 130 & - \\
\hline
\end{tabular}

MDRs/MRPs, multidrug resistance/multidrug resistance-associated proteins.

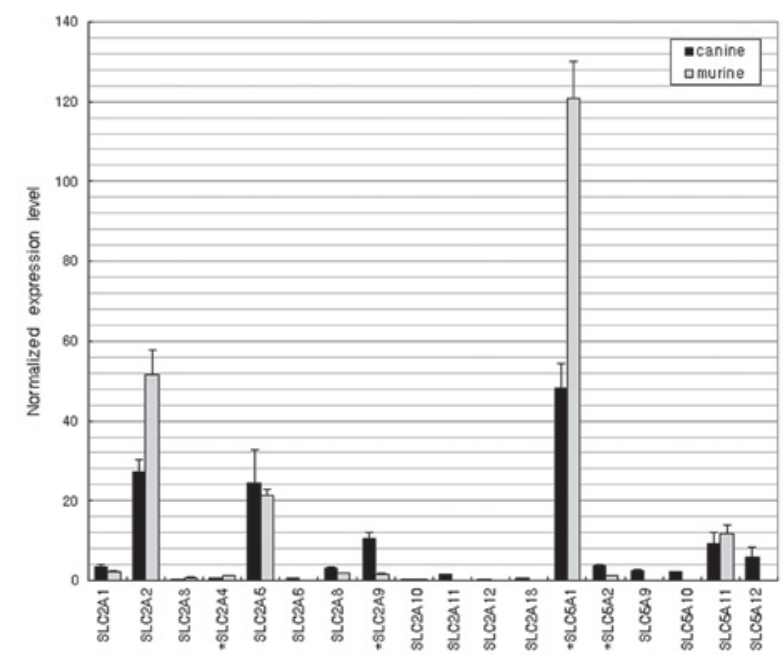

Figure 1. Comparative gene expression of various glucose transporters in the intestines of dogs and mice $(n=4)$. Expression levels were measured as the intensity of the hybridization signal in a GeneChip array and normalized using GeneSpring 7.2. * $>2$-fold difference in gene expression between the two species (t-test, $\mathrm{P}<0.01$ ). SLC2, solute carrier family 2 (GLUTs, facilitated glucose transporters); SLC5, solute carrier family 5 (SGLTs, sodium/glucose cotransporters).

levels of fatty acid transporter genes were relatively high compared with those of the other transporter groups (Table I). Among the fatty acid binding proteins (FABPs), FABP1 and FABP2 were the most dominantly expressed in both species.

Nucleobase and nucleoside transporters. As nucleobase transporters, SLC23A1 and SLC23A2 were highly expressed in mice but poorly expressed in dogs (Fig. 4). Among the nucleoside transporter genes, SLC28A2 (a sodium-coupled

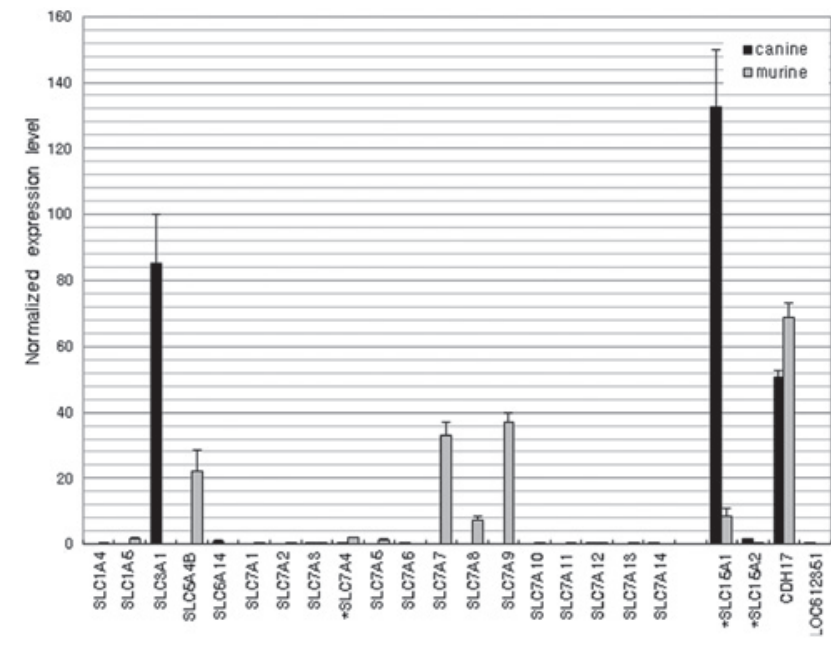

Figure 2. Comparative expression of various amino acid and peptide transporter genes in the intestines of dogs and mice $(n=4)$. SLC1, solute carrier family 1 (neuronal/epithelial high affinity glutamate transporters, system Xag); SLC5, solute carrier family 5 (sodium/glucose cotransporters); SLC7, solute carrier family 7 (cationic amino acid transporters, y+ system); CDH17, cadherin 17/Hpt1, SLC15 solute carrier family 15 (oligopeptide transporters); LOC612351, similar to peptide/histidine transporter PHT2.

nucleoside transporter) was highly expressed in mice but not in dogs. Another family of nucleoside transporters, including SLC29A1 and SLC29A2, was more highly expressed in dogs than in mice.

$M D R$ and MRPs. Fig. 5 shows the expression levels of ATP-binding cassette (ABC) genes. Similarly high expression of ABCB1A (MDR/TAP) and ABCC2 (CFTR/MRP) were observed in both species. 


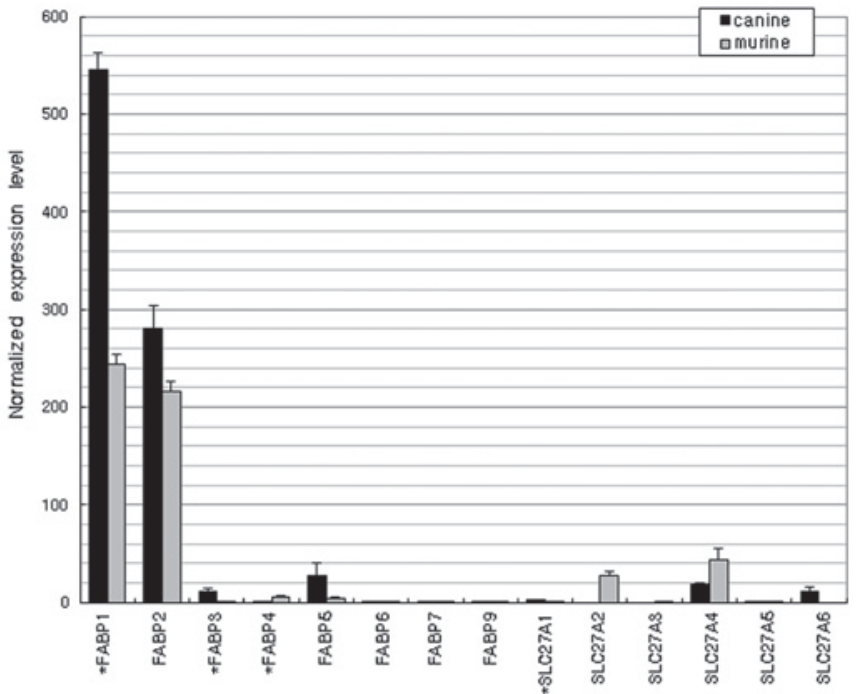

Figure 3. Comparative expression of various fatty acid transporter genes in the intestines of dogs and mice $(\mathrm{n}=4)$. FABP, fatty acid binding protein; SLC27, solute carrier family 27 (fatty acid transporters).

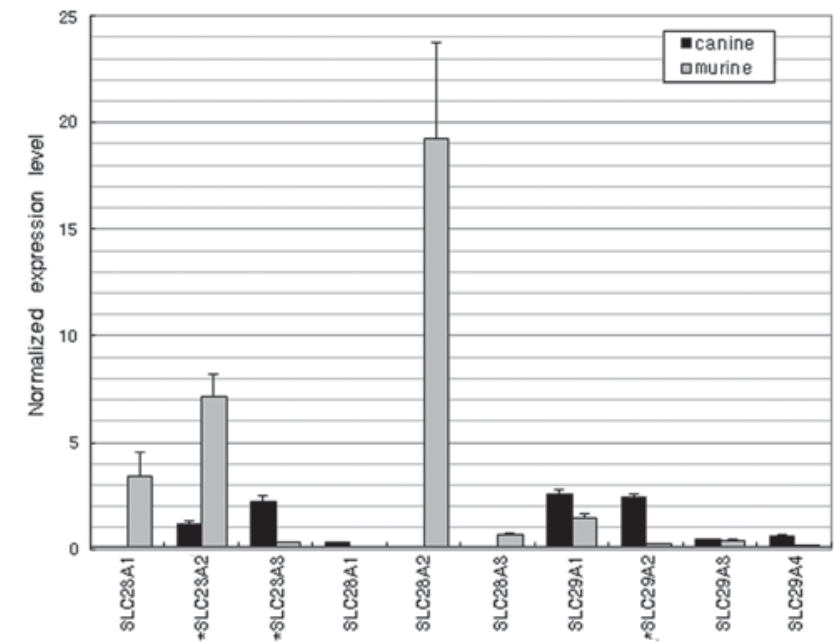

Figure 4. Comparative expression of various nucleobase and nucleoside transporter genes in the intestines of dogs and mice $(n=4)$. SLC23, solute carrier family 23 (nucleobase transporters); SLC28, solute carrier family 28 (sodium-coupled nucleoside transporters, CNTs); SLC29, solute carrier family 29 (nucleoside transporters).

Organic anion and cation transporters. Fig. 6 shows an interspecies variation in the expression levels of organic anion transporters. The most highly expressed anion transporter genes in the dog duodenum were SLC22A9 and SLCO4A1 and the most strongly expressed gene in mice was SLCO2A1. Of the organic cation transporters (Fig. 7), SLC22A1, SLC22A5 and SLC22A18 were dominantly expressed in the mice although their expression levels were negligible in the dogs. However, the expression of SLC22A13 in dogs was $>30$-fold higher than that in mice $(\mathrm{P}<0.01)$.

Phosphate transporters. Among the various phosphate transporters, the SLC17 (sodium phosphate transporter), SLC20 (phosphate transporter), SLC34 (sodium phosphate transporter) and SLC37 (glycerol-3-phosphate transporter) family

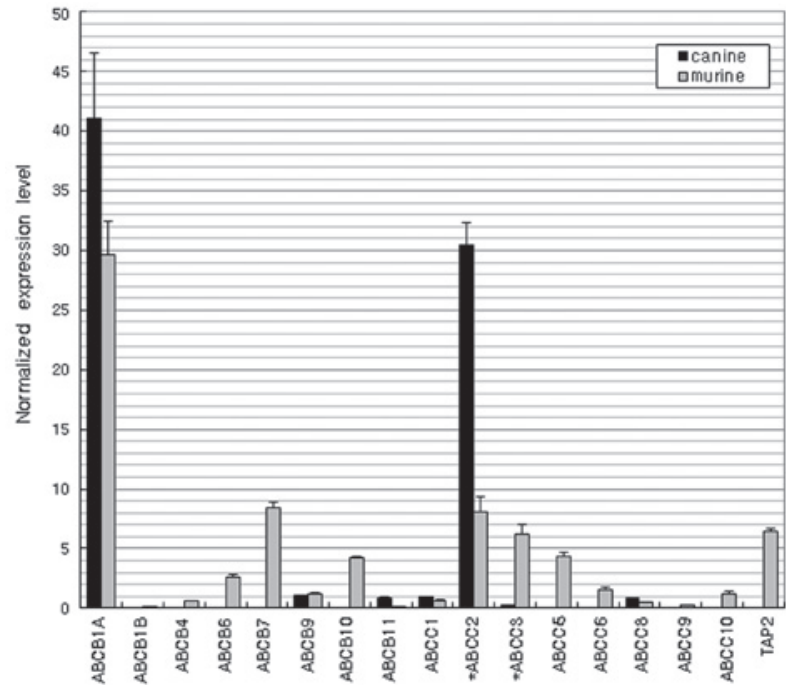

Figure 5. Comparative expression of various MDR and MRP genes in the intestines of dogs and mice $(n=4)$. MDR/MRP, multidrug resistance/multidrug resistance-associated protein; ABCB, ATP-binding cassette sub-family B (MDR/TAP); ABCC, ATP-binding cassette sub-family C (CFTR/MRP); TAP2, transporter 2 ATP-binding cassette sub-family B (MDR/TAP).

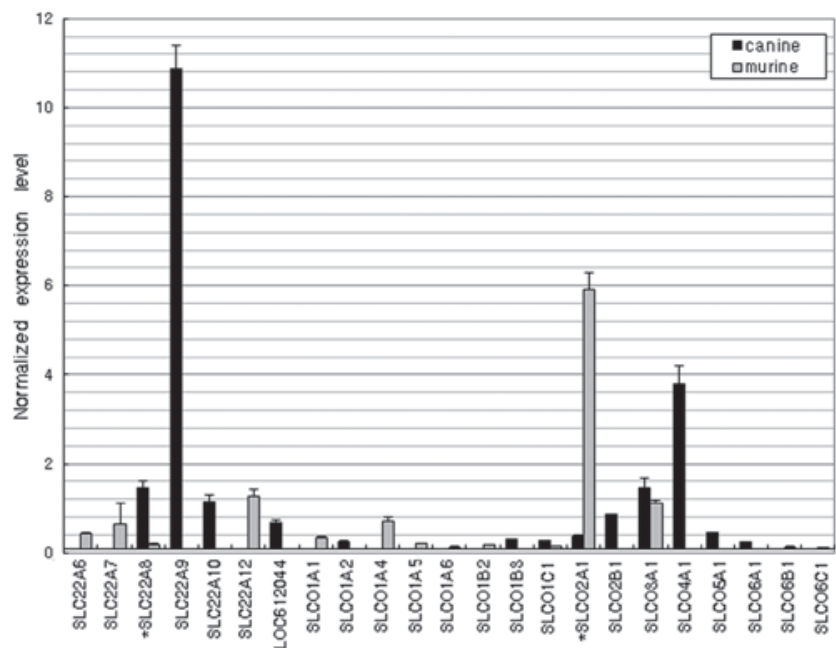

Figure 6. Comparative expression of various organic anion transporter genes in the intestines of dogs and mice $(n=4)$. SLC22, solute carrier family 22 (organic cation transporters); LOC612044, similar to SLC22A20; SLCO, solute carrier organic anion transporter family.

genes were detected in the dogs (Fig. 8). Only 4 SLC37 family genes, SLC37A1, SLC37A2, SLC37A3 and SLC37A4, were expressed in the mice. The most highly expressed genes were SLC20A1 in dogs and SLC37A4 in mice $(\mathrm{P}<0.01)$.

\section{Discussion}

The completion of the DNA sequencing of the human, mouse, dog and rat genomes and knowledge of cross-species gene homologies enables the study of differential gene expression in animal models (7). The characterization of tissue-specific genes, such as intestinal transporters, has the potential to greatly enhance the understanding of the bioavailability of oral drugs (8-13). It is now accepted that the process of drug absorption in the intestine is highly associated with the func- 


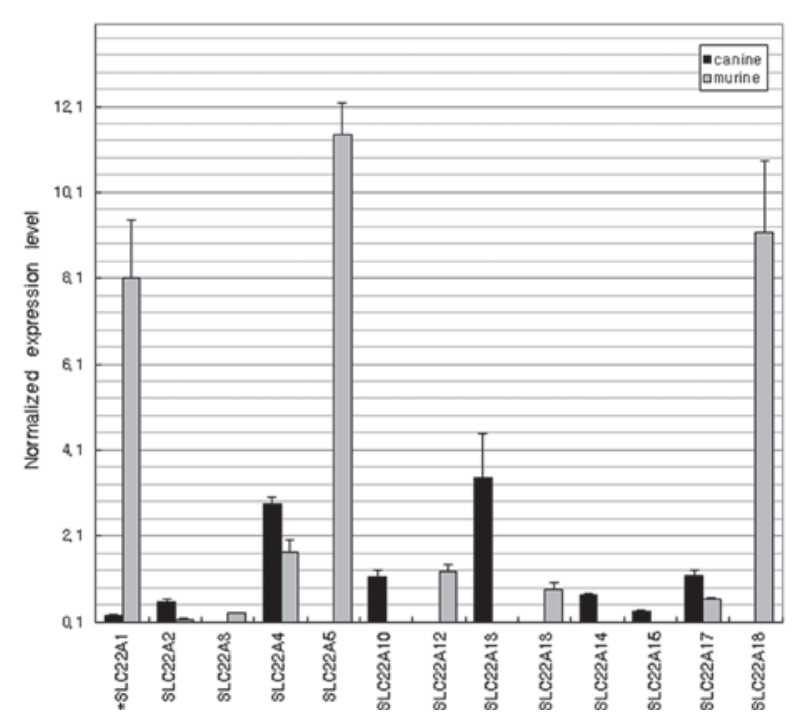

Figure 7. Comparative expression of various organic cation transporter genes in the intestines of dogs and mice $(n=4)$. SLC22, solute carrier family 22 (organic cation transporters).

tional gene expression of intestinal transporters (8). However, little is known about the transporter genes that are transcribed in the dog intestine.

Membrane transport is a critical process for the intestinal absorption of glucose, one of the primary energy sources for various physiological and pharmacological functions (14). All glucose transporters have significant homologies but they function differently to transport various sugars or sugar-associated compounds from the intestine to the systemic circulation with interspecies diversities (15). The data of the present study revealed that the three glucose transporters SLC2A2 (GLUT2), SLC2A5 (GLUT5) and SLC5A1 (SGLT1) are similarly dominantly expressed in dogs and mice. In humans, SLC5A1 transports glucose from the intestinal lumen to the cytosol and SLC2A2 transports glucose from the cytosol to the blood. Intestinal glucose absorption by the apical SLC2A2 pathway may be 3- to 5-times greater than that by SGLT1 at high concentrations of sugar (16). SLC2A5 preferentially transports fructose rather than glucose (17).

The classification of amino acid transporters is well defined according to their substrate specificity and tissue distribution (18). The amino acid transporters have highly restrictive substrate specificities (19). The intestinal uptake of certain anticancer drugs, such as the toxicants gabapentin, pregabalin, melphalan, baclofen, D-cycloserine and methylmercury L-cysteine complex, and other drugs is known to be mediated specifically by these transporters. In the present study, a significant difference was observed between the expression of the transporters in the two species. SLC3A1 was dominantly expressed in dogs and SLC7A9 and SLC7A7 (cationic amino acid transporters) were detected at high levels in mice. Previous studies have demonstrated that amino acid transporters are good targets for improving the oral bioavailability of amino acid-associated drugs. The peptide transporter SLC15A1 (PEPT1) was expressed in both species but its expression level was 15 -fold higher in dogs than in mice. The proton-coupled peptide transporter is responsible for the absorption of small peptides arising from the diges-

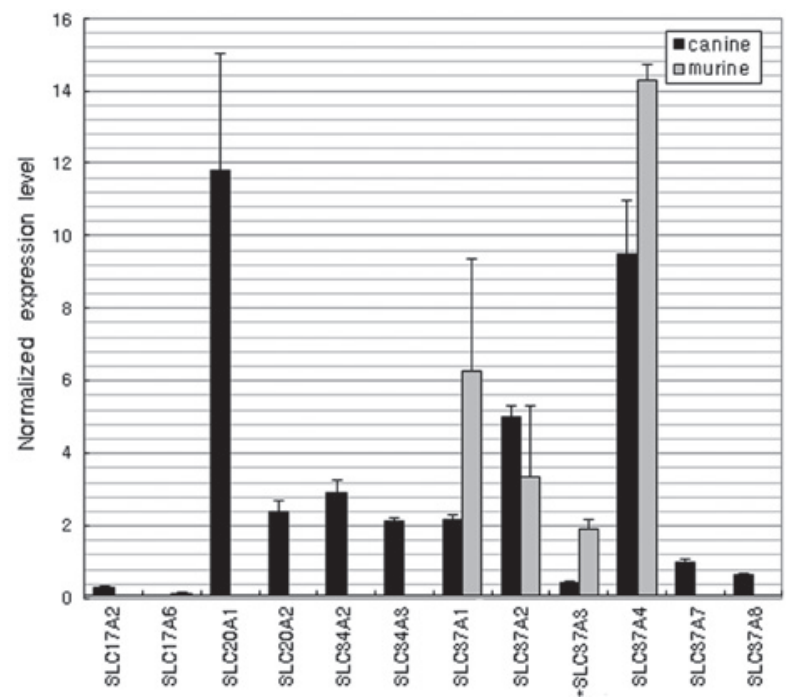

Figure 8. Comparative expression of various phosphate transporter genes in the intestines of dogs and mice $(n=4)$. SLC17, solute carrier family 17 (sodium phosphate transporters); SLC20, solute carrier family 20 (phosphate transporters); SLC34, solute carrier family 34 (sodium phosphate transporters); SLC37, solute carrier family 37 (glycerol-3-phosphate transporters).

tion of dietary proteins (20). It is well known that SLC15A1 is responsible for the uptake of a number of peptide-like drugs, including $\beta$-lactam antibiotics, angiotensin converting enzyme inhibitors, renin inhibitors, antitumor or antiviral agents, thrombin inhibitors, a dopamine receptor antagonist and amino acid prodrugs (21). Kim et al (22) also reported the notable expression of SLC15A1 in the rat intestine.

Fatty acids are absorbed by passive diffusion. However, numerous studies have demonstrated that various transporters are involved in fatty acid absorption (23). Intestinal enterocytes contain high concentrations of two cytosolic FABPs, FABP1 and FABP2, which are hypothesized to be involved in cellular fatty acid trafficking. It was suggested that FABP2, but not FABP1, may directly extract fatty acids from membranes (24). The present results reveal that the two FABP genes are the principal fatty acid transporters expressed in the intestines of dogs and mice with similar expression trends in both species. Schaffer and Lodish (25) identified a membrane protein which they termed fatty acid transport protein (FATP) from mouse adipocytes. However, in the present study, the expression of FATP was not particularly strong in either species.

Aside from the crucial roles of purines and pyrimidines in DNA and RNA synthesis, they are also significant components of a number of important biomolecules (26). Therefore, there is a specific transport system associated with active transporters for delivering nucleobases or nucleosides into cells. In the present study, a considerable difference in the expression of nucleobase and nucleoside transporters was observed between dogs and mice. In mice, the nucleobase transporters SLC23A1 and SLC23A2 and nucleoside transporter SLC28A2 (sodium-coupled nucleoside transporters) were highly expressed but these genes were poorly expressed in dogs. By contrast, the nucleoside transporters SLC29A1 and SLC29A2 were highly expressed in dogs. Nucleobase and nucleoside analogs are widely used in the treatment of neoplasms and viral infections $(27,28)$. 
Typical efflux transporters involve MRPs and MDRs, which are members of the ABC superfamily. These proteins translocate a wide variety of substrates, including sugars, amino acids, nucleosides, lipids, bile salts, metal ions, peptides, proteins and a large number of hydrophobic compounds and metabolites across extra- and intracellular membranes $(29,30)$. In the present study, ABCB1A and ABCC2 (MRP2) were the most highly expressed genes in the two species. Previous studies revealed that $\mathrm{ABCC} 2$ accepts glutathione conjugates, sulfate conjugates, glucuronides and non-conjugated organic anions, pravastatin, vinblastine, temocaprilat, BQ-123, methotrexate, irinotecan and HIV protease inhibitors, including saquinavir, ritonavir and indinavir. ABCC3 (MRP3) mediates the transport of glucuronide conjugates, taurocholate, glycocholate and methotrexate. Differential expression of ABCC3 was observed between the two species but the expression levels of $\mathrm{ABCC} 3$ were lower than those of $\mathrm{ABCC} 2$.

Organic anion transporters serve as the efflux system for a number of endogenous compounds, anionic drugs, environmental substances and their metabolic products, which are usually harmful to the body. In particular, these families are known to affect the pharmacokinetics and drug-drug interactions of various drugs (31). In the present study, SLC22A9 and SLCO4A1 in dogs and SLCO2A1 in mice were dominantly expressed and the overall levels of expression were higher in dogs than in mice. SLCO4A1 mediates the sodium-independent transport of organic anions, including the thyroid hormones triiodo-L-thyronine and thyroxine. Organic cation transporters in the liver, kidney and intestine are critical for the absorption and elimination of a number of endogenous amines as well as a wide range of drugs and environmental toxins. In the present study, the SLC22 family gene expression profile was markedly different between the dogs and mice. The expression of SLC22A13 was more than 30-fold higher in dogs than in mice. SLC22A13 was originally known as an organic cation transporter but Bahn et al (32) renamed it OAT10 (organic anion transporter) since it is able to transport various anionic compounds, including nicotinate, $\mathrm{p}$-aminohippurate and urate.

In the analysis of phosphate transporters, the data reveal that members of the SLC17, SLC20, SLC34 and SLC37 families were expressed in the dogs, while only SLC37 family genes, including SLC37A1, SLC37A2, SLC37A3 and SLC37A4, were expressed in the mice. The most highly expressed genes in the dogs and mice were SLC20A1 and SLC37A4, respectively. SLC20A1 is a ubiquitously expressed sodium-phosphate symporter that plays a fundamental housekeeping role in the maintenance of phosphate homeostasis. SLC20A1 may also function as a retroviral receptor as it confers susceptibility to certain viral infections in human cells.

Although similar profiles were observed for several transporters in both species, including the glucose transporters SLC5A1 and SLC2As, the fatty acid transporters FABP1 and FABP2, the efflux transporters $\mathrm{ABCB} 1 \mathrm{~A}$ and $\mathrm{ABCC} 2$ and the phosphate transporter SLC37A4, overall, the data of the present study reveal markedly different transcriptomic profiles for the intestinal transporters of dogs and mice. The dog is an extremely important animal species, not only as a laboratory animal, but also as a major animal in veterinary medicine. However, genetic information has not been explored exten- sively for the dog in comparison with other model animals. Therefore, the database generated in the present study may be useful for comparing the intestinal xenobiotic transport systems of dogs and other animals.

\section{Acknowledgements}

This study was supported by a grant (10162KFDA995) from the Korea Food \& Drug Administration in 2012.

\section{References}

1. Varma MV: Role of intestinal transporters and metabolism in the oral absorption of drug and prodrugs. Curr Drug Metab 11: $715,2010$.

2. Katsura $\mathrm{T}$ and Inui K: Intestinal absorption of drugs mediated by drug transporters: mechanisms and regulation. Drug Metab Pharmacokinet 18: 1-15, 2003.

3. Sugiura T, Kato Y and Tsuji A: Role of SLC xenobiotic transporters and their regulatory mechanisms PDZ proteins in drug delivery and disposition. J Control Release 116: 238-246, 2006.

4. Rankin KS, Starkey M, Lunec J, et al: Of dogs and men comparative biology as a tool for the discovery of novel biomarkers and drug development targets in osteosarcoma. Pediatr Blood Cancer 58: 327-333, 2012.

5. Wang W, Jiang J, Ballard CE and Wang B: Prodrug approaches to the improved delivery of peptide drugs. Curr Pharm Des 5: 265-287, 1999.

6. Shin HC, Kim HR, Cho HJ, et al: Comparative gene expression of intestinal metabolizing enzymes. Biopharm Drug Dispos 30: 411-421, 2009.

7. Fang H, Tong W, Perkins R, et al: Bioinformatics approaches for cross-species liver cancer analysis based on microarray gene expression profiling. BMC Bioinformatics 6 (Suppl 2): S6, 2005.

8. Landowski CP, Sun D, Foster DR, et al: Gene expression in the human intestine and correlation with oral valacyclovir pharmacokinetic parameters. J Pharmacol Exp Ther 306: 778-786, 2003.

9. Takara K, Ohnishi N, Horibe S and Yokayama T: Expression profiles of drug-metabolizing enzyme CYP3A and drug efflux transporter multidrug resistance 1 subfamily mRNAS in small intestine. Drug Metab Dispos 31: 1235-1239, 2003.

10. Rushmore TH and Kong AN: Pharmacogenomics, regulation and signaling pathways of phase I and II drug metabolizing enzymes. Curr Drug Metab 3: 481-490, 2002.

11. Mizuno N, Niwa T, Yotsumoto Y and Sugiyama Y: Impact of drug transporter studies on drug discovery and development. Pharmacol Rev 55: 425-461, 2003.

12. Beaumont K: The importance of gut wall metabolism in determining drug bioavailability. In: Drug Bioavailability/Estimation of Solubility, Permeability and Absorption. van de Waterbeemd $\mathrm{H}$, Lennernas $\mathrm{H}$ and Artursson P (eds). Wiley-VCH, Weinheim, pp311-328, 2003.

13. Tsuji A: Transporter-mediated drug interactions. Drug Metab Pharmacokinet 17: 253-274, 2002.

14. Mizuma T, Ohta K and Awazu S: The beta-anomeric and glucose preferences of glucose transport carrier for intestinal active absorption of monosaccharide conjugates. Biochim Biophys Acta 1200: 117-122, 1994.

15. Zhao FQ and Keating AF: Expression and regulation of glucose transporters in the bovine mammary gland. J Dairy Sci 90 (Suppl 1): E76-E86, 2007.

16. Stelmańskan E: The important role of GLUT2 in intestinal sugar transport and absorption. Postepy Biochem 55: 385-387, 2009 (In Polish).

17. Phay JE, Hussain HB and Moley JF: Cloning and expression analysis of a novel member of the facilitative glucose transporter family, SLC2A9 (GLUT9). Genomics 66: 217-220, 2000.

18. Tate BJ, Witort E, McKenzie IF and Hogarth PM: Expression of the high responder/non-responder human Fc gamma RII. Analysis by PCR and transfection into FCR-COS cells. Immunol Cell Biol 70 (Pt 2): 79-87, 1992.

19. Wells RG and Hediger MA: Cloning of a rat kidney cDNA that stimulates dibasic and neutral amino acid transport and has sequence similarity to glucosidases. Proc Natl Acad Sci USA 89: 5596-5600, 1992. 
20. Seal CJ and Parker DS: Isolation and characterization of circulating low molecular weight peptides in steer, sheep and rat portal and peripheral blood. Comp Biochem Physiol B 99: 679-685, 1991.

21. Lee VH, Chu C, Mahlin ED, et al: Biopharmaceutics of transmucosal peptide and protein drug administration: role of transport mechanisms with a focus on the involvement of PepT1. J Control Release 62: 129-140, 1999.

22. Kim HR, Park SW, Cho HJ, et al: Comparative gene expression profiles of intestinal transporters in mice, rats and humans. Pharmacol Res 56: 224-236, 2007.

23. Abumrad N, Harmon C, and Ibrahimi A: Membrane transport of long-chain fatty acids: evidence for a facilitated process. J Lipid Res 39: 2309-2318, 1998.

24. Thumser AE and Storch J: Liver and intestinal fatty acid-binding proteins obtain fatty acids from phospholipid membranes by different mechanisms. J Lipid Res 41: 647-656, 2000 .

25. Schaffer JE and Lodish HF: Expression cloning and characterization of a novel adipocyte long chain fatty acid transport protein. Cell 79: 427-436, 1994.
26. Traut TW: Physiological concentrations of purines and pyrimidines. Mol Cell Biochem 140: 1-22, 1994.

27. el Kouni MH: Trends in the design of nucleoside analogues as anti-HIV drugs. Curr Pharm Des 8: 581-593, 2002.

28. Damaraju VL, Damaraju S, Young JD, et al: Nucleoside anticancer drugs: the role of nucleoside transporters in resistance to cancer chemotherapy. Oncogene 22: 7524-7536, 2003.

29. Sandusky GE, Mintze KS, Pratt SE and Dantzig AH: Expression of multidrug resistance-associated protein 2 (MRP2) in normal human tissues and carcinomas using tissue microarrays. Histopathology 41: 65-74, 2002.

30. Tang F and Borchardt RT: Characterization of the efflux transporter(s) responsible for restricting intestinal mucosa permeation of an acyloxyalkoxy-based cyclic prodrug of the opioid peptide DADLE. Pharm Res 19: 780-786, 2002.

31. Miyazaki H, Sekine T and Endou H: The multispecific organic anion transporter family: properties and pharmacological significance. Trends Pharmacol Sci 25: 654-662, 2004.

32. Bahn A, Hagos Y, Reuter S, et al: Identification of a new urate and high affinity nicotinate transporter, hOAT10 (SLC22A13). J Biol Chem 283: 16332-16341, 2008. 